\title{
ESTUDO CLÍNICO E VIDEOFLUOROSCÓPICO DA DISFAGIA NA FASE SUBAGUDA DO ACIDENTE VASCULAR ENCEFÁLICO*
}

\author{
Denise Rodrigues Xerez ${ }^{1}$, Yonatta Salarini Vieira Carvalho ${ }^{2}$, Milton Melciades Barbosa Costa ${ }^{3}$
}

Resumo OBJETIVO: Correlacionar, em pacientes portadores de acidente vascular encefálico (AVE) na fase subaguda, as alterações clínicas da deglutição com as observadas na videofluoroscopia. MATERIAIS E MÉTODOS: De 37 portadores de AVE subagudo confirmado por exame de imagem, 26 pacientes de ambos os sexos, com idade média de 59,69 anos, foram avaliados clínica e videofluoroscopicamente. Consideramos como variáveis para pareamento estatístico os parâmetros clínicos indicativos de penetração/aspiração e sua confirmação na videofluoroscopia. RESULTADOS: Identificamos disfagia em 19 (73\%) dos 26 pacientes que fizeram videofluoroscopia; dez $(38,46 \%)$ apresentaram penetração/aspiração de líquidos. Os dados resultantes mostraram não existir correlação $(p<0,05)$ entre a presença de disfagia e/ou disartria e a presença de penetração/aspiração de líquidos na videofluoroscopia. Houve correlação entre a presença de penetração/aspiração de líquidos observados na videofluoroscopia com os seguintes parâmetros clínicos: estado dos dentes ( $p=0,047)$, mobilidade $(p=0,019)$ e sensibilidade da face $(p=0,039$ ) e mobilidade da língua ( $p=0,012$ ). CONCLUSÃO: Não foi possível definir a presença de penetração/aspiração de líquidos nas vias aéreas por dados epidemiológicos do AVE. A existência de mau estado de preservação dos dentes, alterações na mobilidade da face e da língua e na sensibilidade da face mostrou risco aumentado de penetração/ aspiração para líquidos. Permanece importante a indicação da videofluoroscopia para melhor avaliação dos quadros de disfagia após AVE.

Unitermos: Disfagia; Acidente vascular encefálico; Pneumonia aspirativa; Videofluoroscopia; Radiologia; Reabilitação.

Abstract Clinical and videofluoroscopic study of dysphagia in patients with cerebrovascular accident in the subacute phase.

OBJECTIVE: To compare the clinical signs of swallowing abnormalities with videofluoroscopy findings in patients with cerebrovascular accident (CVA) in the subacute phase. MATERIALS AND METHODS: From a group of 37 patients with subacute CVA confirmed by imaging examinations, 26 patients (male and female; mean age of 59.69 years) were clinically and videofluoroscopically evaluated. The clinical parameters for penetration/aspiration of fluids and the confirmation by videofluoroscopy were used for the statistical analysis. RESULTS: Dysphagia was identified in 19 (73\%) of the 26 patients who were submitted to videofluoroscopy; $10(38.6 \%)$ patients presented penetration/aspiration of fluids. The results demonstrated that there was no correlation $(p<0.05)$ between the occurrence of dysphagia and/or dysartria and penetration/aspiration of fluids observed on videofluoroscopy. There was correlation between penetration/aspiration of liquids observed on videofluoroscopy and the following parameters: condition of the teeth $(p=$ 0.047 ), face motility ( $p=0.019)$ and sensibility $(p=0.039)$, and tongue motility $(p=0.012)$. CONCLUSION: It was not possible to determine the presence of penetration/aspiration of liquids in the airways by the epidemiological data of the CVA. Patients with bad teeth, facial and/or tongue motility abnormalities, or facial sensibility showed increased risk of penetration/aspiration of liquids. Videofluoroscopic assessment remains an important means to better evaluate dysphagia in patients with CVA.

Key words: Dysphagia; Cerebrovascular accident; Aspiration pneumonia; Videofluoroscopy; Radiology; Rehabilitation.

\footnotetext{
* Trabalho realizado em laboratórios do Centro de Ciências da Saúde da Universidade Federal do Rio de Janeiro (UFRJ), Rio de Janeiro, RJ.

1. Médica Fisiatra, Mestre em Medicina, Professora Assistente da Faculdade de Medicina da UFRJ.

2. Fonoaudióloga, Mestranda do Programa de Pós-Graduação em Ciências do Departamento de Clínica Médica da Faculdade de Medicina da UFRJ.

3. Médico, Doutor em Medicina, Professor Titular de Anatomia do Instituto de Ciências Biológicas da UFRJ.

Endereço para correspondência: Profa. Dra. Denise Rodrigues Xerez. Serviço de Medicina Física e Reabilitação - HUCFF-UFRJ. Avenida Brigadeiro Trompowski, s/no , Cidade Universitária. Rio de Janeiro, RJ, 21941-590. E-mail: xerez@uol.com.br

Recebido para publicação em 19/3/2003. Aceito, após revisão, em 26/5/2003.
}

\section{INTRODUÇÃO}

Os acidentes vasculares encefálicos (AVE) são a maior causa de deficiência motora adquirida, sendo responsáveis por até $80 \%$ das incapacidades e a terceira causa de morte em adultos ${ }^{(1)}$

Está descrita a ocorrência de 700.000 casos de AVE anuais, sendo 500.000 novos e 200.000 recorrentes, totalizando, na população mundial, aproximadamente 3,89 milhões de indivíduos sobreviventes a esta afecção ${ }^{(2)}$.

Segundo referem Lawrence et al., mais da metade dos pacientes $(50,6 \%)$ tem entre seis e dez tipos de incapacidade, sendo a mais prevalente a fraqueza muscular, presente em $77,4 \%$ dos pacientes, seguida dos distúrbios da comunicação e linguagem e da disfagia, esta última figurando em terceiro lugar, com $44,7 \%$ das incapacidades encontradas ${ }^{(3)}$. 
A disfagia relacionada ao AVE, presente em quase metade dos casos, não é exclusiva das lesões de mesencéfalo e dos dois hemisférios simultaneamente, como classicamente acreditado $^{(4)}$, mas pode ocorrer em todas as formas desses acidentes ${ }^{(\mathbf{5})}$.

A aspiração associada ao AVE tem sido admitida como responsável por três vezes mais casos de pneumonia que as observadas em pacientes acamados por outras causas. Acredita-se que isso se deva à aspiração, algumas vezes silenciosa, relacionada à disfagia resultante da lesão encefálica ${ }^{(4,5)}$.

Há protocolos e recomendações ${ }^{(\mathbf{4 , 6}, 7)}$ voltados para a avaliação e manuseio dos sobreviventes ao AVE. Nesses documentos, a disfagia está elencada como causa de complicações e responsável pelo empobrecimento do prognóstico, em razão da possível ocorrência de broncopneumonia, má nutrição e desidratação. Apesar da evidente importância dos protocolos de avaliação rotineira da dinâmica da deglutição nesta população, não encontramos, em nosso meio, qualquer rotina de identificação e prevenção das complicações das disfagias nas vítimas de AVE. Por esta razão, elaboramos e testamos protocolo clínico para correlacionar as alterações da dinâmica da deglutição com aquelas observadas na avaliação videofluoroscópica.

\section{MATERIAIS E MÉTODOS}

Avaliamos, clinicamente, 37 pacientes (Tabela 1) portadores de AVE, com idades entre 22 e 81 anos $(60 \pm 13,01)$, sendo 17 do sexo feminino e 20 do sexo masculino. Estes pacientes foram encaminhados ao Serviço de Medicina Física e Reabilitação do Hospital Universitário Clementino Fraga Filho (HUCFF) da Universidade Federal do Rio de Janeiro, para tratamento global em reabilitação, entre janeiro de 2000 e dezembro de 2001.

Utilizamos como critério de inclusão o paciente ter sofrido AVE num período de 30 a 365 dias e diagnóstico confirmado por exame de imagem (tomografia computadorizada ou ressonância magnética). Como critério de exclusão observamos estado de coma e agitação psicomotora grave.

Todos os pacientes $(n=37)$ foram avaliados clinicamente, valorizando-se os parâmetros referidos na Tabela 2 .

Tabela 1 Características do grupo.

\begin{tabular}{|c|c|}
\hline Total de pacientes: & $37(100 \%)$ \\
\hline Pacientes com VFC: & $26(70,27 \%)$ \\
\hline Sexo: & Masculino $=20$ (54,05\%); Feminino = $17(45,95 \%)$ \\
\hline Dominância: & $\operatorname{Sim}=14(37,84 \%) ;$ Não = $23(62,15 \%)$ \\
\hline Tempo AVE (dias): & De 30 a 360 (média de 104,32; desvio padrão de 97,51) \\
\hline Idade (anos): & De 22 a 81 (média de 60,95; desvio padrão de 13,01) \\
\hline Disfagia: & $\begin{array}{l}\text { Não }=9(24,32 \%) ; \text { Leve = } 11(29,73 \%) ; \text { Moderada = } 10(27,03 \%) ; \text { Grave } \\
=7(18,92 \%)\end{array}$ \\
\hline Grau de hemiplegia: & Leve = 19 (51,35\%); Moderada = $11(29,73 \%) ;$ Grave $=7(18,92 \%)$ \\
\hline Alimentação: & $\begin{array}{l}\text { Sonda nasogástrica }=2(5,40 \%) ; \text { Gastrostomia }=3(8,11 \%) ; \text { Normal }=32 \\
(86,49 \%)\end{array}$ \\
\hline Exame clínico oral: & Normal $=0(0 \%) ;$ Alterado = $37(100 \%)$ \\
\hline Exame clínico faringe: & Normal = 17 (45,95\%); Alterado = 20 (54,05\%) \\
\hline VFC oral: & Normal = $0(0 \%) ;$ Alterado = $26(100 \%)$ \\
\hline VFC faringe: & Normal = $10(38,46 \%) ;$ Alterado = $16(61,54 \%)$ \\
\hline
\end{tabular}

VFC, videofluoroscopia; AVE, acidente vascular encefálico.

Tabela 2 Parâmetros clínicos.

\begin{tabular}{|l|l|}
\hline \multicolumn{1}{|c|}{ Parâmetro } & \multicolumn{1}{c|}{ Classificação do parâmetro } \\
\hline $\begin{array}{l}\text { Presença de disfagia } \\
\text { Presença de disartria }\end{array}$ & Sim/não \\
Preparo e qualificação & Sim/não \\
Estado dos dentes/prótese & Normal (bom estado)/alterado (mau estado ou \\
Mobilidade da face & ausentes) \\
Sensibilidade da face & Normal/alterado \\
Oral & Normal/alterado \\
Mobilidade da língua & \\
Gustação & Normal/alterado \\
Mobilidade do palato & Normal/alterado \\
Reflexo nauseoso & Normal/alterado \\
Faríngea & Normal/alterado \\
Mobilidade do hióide & \\
Presença de penetração/aspiração de líquido & Sim/não \\
\hline
\end{tabular}

Vinte e seis pacientes foram submetidos à videofluoroscopia. Foram utilizados meios de contraste nas consistências liíquida, sólida e pastosa ${ }^{(8)}$, com intervalo de até sete dias da avaliação clínica, em equipamento de RX Medicor UV 56M, tipo FR2, com tubo D19-12/50-150, com sistema de TV Videomed 2 com intensificador de imagem tipo RBV 12/13, tubo tipo Vidicon, padrão 525 linhas, $60 \mathrm{~Hz}$, entrelaçamento $2: 1$. As imagens foram registradas em fita VHS, com sistema vídeo/monitor Samsung, modelo-CXE 1331.

Os registros videofluoroscópicos foram realizados por meio de vídeo AG 96 Panasonic. Os parâmetros analisados (Tabela 3) foram efetuados por três examinadores e laudados com o programa Sirredid (siste- ma de registro e recuperação de dados da avaliação dinâmica da deglutição) ${ }^{(\mathbf{9})}$.

Todos os pacientes foram esclarecidos dos propósitos do estudo e assinaram termo de consentimento aprovado pelo comitê de ética em pesquisa do HUCFF.

\section{Procedimento estatístico}

Utilizamos o teste não-paramétrico quiquadrado, cruzando par a par todos os parâmetros observados.

Tomou-se como referência decisória, quanto à significância dos resultados, o índice $\mathrm{p}<0,05$, ou seja, foram aceitos resultados que se apresentaram com um mínimo $95 \%$ de certeza.

Uma vez que os dados observados são de natureza discreta, montamos tabelas de 
distribuição de freqüência para análise das possíveis relações funcionais entre os parâmetros considerados.

\section{RESULTADOS}

Identificamos disfagia em $28(75,68 \%)$ dos 37 pacientes. Desses 37, $11(29,73 \%)$ apresentavam quadro leve, dez $(27,03 \%)$ apresentavam disfagia moderada, e sete $(18,92 \%)$, disfagia grave.

Detectamos comprometimento da fase oral durante a avaliação clínica nos 37 indivíduos analisados. A avaliação clínica da fase faríngea nos permitiu a observação de alterações em 20 deles $(54,05 \%)$.

Os 26 pacientes $(54,05 \%)$ avaliados videofluoroscopicamente tiveram confirmada a existência de alteração funcional da fase oral relacionada à observação clínica.

A avaliação clínica da fase faríngea desses 26 pacientes nos permitiu supor que a dinâmica hióidea e laríngea estava alterada, debilitando a proteção das vias aéreas em 14 dos pacientes examinados, e que havia sinais positivos de penetração/aspiração (voz molhada e ausculta cervical típica de aspiração) em 11; não identificamos sinais premonitórios em 15 pacientes.

A avaliação videofluoroscópica dos 14 pacientes com mobilidade hio-laríngea considerada clinicamente alterada deixou ver que sete $(50 \%)$ tiveram penetração das vias aéreas pelo contraste líquido. Dos 12 que não tinham alterações da dinâmica hio-laríngea, três aspiraram. No que se refere aos outros sinais premonitórios de aspiração, dos 11 que apresentavam estes sinais, cinco apresentaram aspiração e seis mostraram proteção adequada das vias aéreas. Dos 15 que não tinham sinais clínicos de aspiração, cinco deixaram ver penetração/aspiração durante o exame videofluoroscópico.

Dos 26 pacientes examinados clínica e videofluoroscopicamente, 14 apresentavam algum sinal clínico suspeito de disfunção orofaríngea. Videofluoroscopicamente, 16 deixaram ver algum tipo de anormalidade. Dez apresentaram algum grau de penetração/aspiração e seis permitiram a observação de alterações estruturais clinicamente insuspeitas.

Na Tabela 4 observamos que nenhum dos parâmetros relativos à identificação e

Tabela 3 Parâmetros videofluoroscópicos.

\begin{tabular}{|l|l|}
\hline \multicolumn{1}{|c|}{ Tipo } & \multicolumn{1}{c|}{ Classificação } \\
\hline $\begin{array}{l}\text { Fase oral } \\
\text { Preparo e qualificação }\end{array}$ & Adequado/inadequado \\
Organização/ejeção & Normal/“dipper”/dois tempos \\
Ejeção & Eficiente/deficiente \\
Escape para a rinofaringe & Sim/não \\
Fase faríngea & \\
Elevação do hióide & Adequado/inadequado \\
Abertura da transição faringoesofágica & Adequado/inadequado \\
Proteção & \\
Penetração & \\
Sólido & Sim/não \\
Líquido & Sim/não \\
Pastoso & Sim/não \\
Aspiração & Sim/não \\
Sólido & Sim/não \\
Líquido & Sim/não \\
Pastoso & $\mathrm{Sim} /$ não \\
Musculatura constritora & \\
Protrusão &
\end{tabular}

Tabela 4 Cruzamento entre presença de penetração/aspiração de líquido e parâmetros relativos à identificação e classificação do AVE.

\begin{tabular}{|c|c|c|c|c|c|}
\hline \multirow{3}{*}{$\begin{array}{l}\text { Parâmetros } \\
\text { de identificação } \\
\text { e classificação }\end{array}$} & \multirow{3}{*}{$P_{\text {calculado }}$} & \multicolumn{4}{|c|}{ Presença de penetração/aspiração de líquido (VFC) } \\
\hline & & \multicolumn{2}{|r|}{ Sim } & \multicolumn{2}{|r|}{ Não } \\
\hline & & $\mathrm{N}$ & $\%$ & $\mathrm{~N}$ & $\%$ \\
\hline Tempo de AVE (meses & \multirow{4}{*}{0,1961} & & & & \\
\hline A (30-119) & & 5 & $50,0 \%$ & 3 & $18,8 \%$ \\
\hline B (120-209) & & 3 & $30,0 \%$ & 10 & $62,5 \%$ \\
\hline C (210-360) & & 2 & $20,0 \%$ & 3 & $18,8 \%$ \\
\hline \multicolumn{6}{|l|}{ Idade (anos) } \\
\hline A $(22-65)$ & \multirow{3}{*}{0,1047} & 4 & $40,0 \%$ & 10 & $62,5 \%$ \\
\hline B $(66-70)$ & & 2 & $20,0 \%$ & 5 & $31,3 \%$ \\
\hline C (71-81) & & 4 & $40,0 \%$ & 1 & $6,3 \%$ \\
\hline \multicolumn{6}{|l|}{ Grau de incapacidade } \\
\hline Leve & \multirow{3}{*}{0,2330} & 5 & $50,0 \%$ & 9 & $56,3 \%$ \\
\hline Moderado & & 2 & $20,0 \%$ & 6 & $37,5 \%$ \\
\hline Grave & & 3 & $30,0 \%$ & 1 & $6,3 \%$ \\
\hline \multicolumn{6}{|l|}{ Dominância } \\
\hline Sim & \multirow{2}{*}{0,3152} & 3 & $30,0 \%$ & 8 & $50,0 \%$ \\
\hline Não & & 7 & $70,0 \%$ & 8 & $50,0 \%$ \\
\hline
\end{tabular}

AVE, acidente vascular encefálico; VFC, videofluoroscopia.

classificação do acidente vascular encefálico apresentou relação de dependência com a presença de penetração/aspiração de líquido.

Pelo teste qui-quadrado (significância $\mathrm{p}<0,05)$ existe relação de dependência entre os dados clínicos relativos ao estado dos dentes $\left(\mathrm{p}_{\text {calculado }}=0,047\right)$, mobilidade da face e da língua $\left(\mathrm{p}_{\text {calculado }}=0,012\right)$ e a sensibilidade da face $\left(\mathrm{p}_{\text {calculado }}=0,039\right)$ com as alterações registradas pelo método videofluoroscópico (Tabela 5 e Figura 1).

\section{DISCUSSÃO}

Smithard et al. ${ }^{(\mathbf{1 0})}$, ao descreverem a história natural da disfagia resultante do AVE, observaram na fase subaguda (28 dias) a incidência de $15 \%$ de aspiração pela videofluoroscopia, e na fase crônica (seis 
Tabela 5 Correlação entre parâmetros clínicos e presença de penetração/aspiração de líquido na videofluoroscopia.

\begin{tabular}{|c|c|c|c|c|c|c|c|c|}
\hline \multirow{3}{*}{ Parâmetro clínico } & \multirow{3}{*}{$P_{\text {calculado }}$} & \multirow{3}{*}{ Classe } & \multicolumn{6}{|c|}{ Penetração/aspiração de líquido } \\
\hline & & & \multicolumn{2}{|c|}{ Sim } & \multicolumn{2}{|c|}{ Não } & \multicolumn{2}{|c|}{ Total } \\
\hline & & & N & $\%$ & $\mathrm{~N}$ & $\%$ & $\mathrm{~N}$ & $\%$ \\
\hline \multirow{2}{*}{ Queixa de disfagia } & \multirow{2}{*}{0,529} & Sim & 8 & $42,1 \%$ & 11 & $57,9 \%$ & 19 & $73,1 \%$ \\
\hline & & Não & 2 & $28,6 \%$ & 5 & $71,4 \%$ & 7 & $26,9 \%$ \\
\hline \multirow{2}{*}{ Queixa de disartria } & \multirow{2}{*}{0,282} & Sim & 6 & $40,0 \%$ & 9 & $60,0 \%$ & 15 & $57,7 \%$ \\
\hline & & Não & 4 & $36,4 \%$ & 7 & $63,6 \%$ & 11 & $42,3 \%$ \\
\hline \multirow{2}{*}{ Estado dos dentes } & \multirow{2}{*}{0,047} & Mau estado & 6 & $31,6 \%$ & 13 & $68,6 \%$ & 19 & $73,1 \%$ \\
\hline & & Bom estado & 4 & $57,1 \%$ & 3 & $42,8 \%$ & 7 & $26,9 \%$ \\
\hline \multirow{2}{*}{ Mobilidade da face } & \multirow{2}{*}{0,019} & Alterado & 6 & $30,0 \%$ & 14 & $70,0 \%$ & 20 & $76,9 \%$ \\
\hline & & Normal & 4 & $66,7 \%$ & 2 & $33,3 \%$ & 6 & $23,1 \%$ \\
\hline \multirow{2}{*}{ Sensibilidade da face } & \multirow{2}{*}{0,039} & Alterado & 2 & $20,0 \%$ & 8 & $80,0 \%$ & 10 & $38,5 \%$ \\
\hline & & Normal & 8 & $50,0 \%$ & 8 & $50,0 \%$ & 16 & $61,5 \%$ \\
\hline \multirow{2}{*}{ Mobilidade da língua } & \multirow{2}{*}{0,012} & Alterado & 3 & $21,4 \%$ & 11 & $78,6 \%$ & 14 & $53,8 \%$ \\
\hline & & Normal & 7 & $58,3 \%$ & 5 & $41,7 \%$ & 12 & $46,2 \%$ \\
\hline \multirow{2}{*}{ Gustação } & \multirow{2}{*}{0,228} & Alterado & 2 & $28,6 \%$ & 5 & $71,4 \%$ & 7 & $26,9 \%$ \\
\hline & & Normal & 8 & $42,1 \%$ & 11 & $57,9 \%$ & 19 & $73,1 \%$ \\
\hline \multirow{2}{*}{ Mobilidade do palato } & \multirow{2}{*}{0,097} & Alterado & 3 & $27,3 \%$ & 8 & $72,7 \%$ & 11 & $42,3 \%$ \\
\hline & & Normal & 7 & $46,7 \%$ & 8 & $53,3 \%$ & 15 & $57,7 \%$ \\
\hline \multirow{2}{*}{ Reflexo nauseoso } & \multirow{2}{*}{0,282} & Alterado & 6 & $40,0 \%$ & 9 & $60,0 \%$ & 15 & $57,7 \%$ \\
\hline & & Normal & 4 & $36,4 \%$ & 7 & $63,6 \%$ & 11 & $42,3 \%$ \\
\hline \multirow{2}{*}{ Mobilidade do hióide } & \multirow{2}{*}{0,217} & Alterado & 7 & $50,0 \%$ & 7 & $50,0 \%$ & 14 & $53,8 \%$ \\
\hline & & Normal & 3 & $25,0 \%$ & 9 & $75,0 \%$ & 12 & $46,2 \%$ \\
\hline \multirow{2}{*}{ Penetração/aspiração de líquido } & \multirow[t]{2}{*}{0,397} & Sim & 5 & $45,5 \%$ & 6 & $54,5 \%$ & 11 & $42,3 \%$ \\
\hline & & Não & 5 & $33,3 \%$ & 10 & $66,7 \%$ & 15 & $57,7 \%$ \\
\hline
\end{tabular}

meses), 6,8\%. O presente estudo mostrou uma incidência de $38,46 \%$ de presença de penetração/aspiração de líquido na videofluoroscopia em pacientes com seqüelas de AVE com tempo médio de instalação de 104,32 dias. Macedo ${ }^{(\mathbf{1 1})}$ encontrou incidência de $66,7 \%$ de penetração na faringe, entretanto não menciona as características do grupo portador desta seqüela. Baseados nesses estudos, os autores recomendaram a identificação do risco de aspiração apenas pela avaliação clínica ${ }^{(12)}$, em divergência com os dados obtidos em nosso estudo, que reforçam a necessidade de avaliação videofluoroscópica.

Daniels et al. $^{(\mathbf{5}, \mathbf{1 3})}$ e Roth e Harvey(2) descrevem e ressaltam fatores epidemiológicos como prognósticos para a recuperação funcional pós-AVE. A idade do paciente e a gravidade da incapacidade motora teriam relação direta com a recuperação motora, isto é, quanto maior a idade e o grau de incapacidade, menor o grau de recuperação funcional. A idade tem-se mostrado um fator de risco para alterações da deglutição por si; Kendall e Leonard ${ }^{(\mathbf{1 4})}$, em es- tudo de um grupo de idosos (maiores de 65 anos), encontraram aumento do tempo de preparo oral como fator característico. Não foi possível observar correlação significativa entre os fatores prognósticos estudados e a ocorrência de penetração/aspiração de líquidos na videofluoroscopia; porém, a descrição da população relacionada às características desta doença é fortemente recomendada para trabalhos com este grupo ${ }^{(15)}$. A lateralidade e dominância do hemisfério afetado, e o tipo de adaptação oral observados foram valorizados. Não observamos correlação entre estes parâmetros e o risco de penetração/aspiração de líquidos. Hamdy et al. ${ }^{(\mathbf{1 6})}$ descrevem a representação de áreas da deglutição em ambos os hemisférios cerebrais e defendem o aumento da atividade no hemisfério são. Muitas das seqüelas observadas na fase aguda do AVE tendem a regredir com o decorrer do tempo, até em média seis meses após o acidente, durante a fase subaguda. Em relação à disfagia, isto é particularmente verdadeiro, já que está descrita resolução espontânea em mais da metade dos $\operatorname{casos}^{(\mathbf{1 0})}$.
Aviv et al. ${ }^{(4)}$ e Roth e Harvey ${ }^{(2)}$ descrevem, em seus estudos, um grande porcentual de ocorrência de aspiração silenciosa em casos de acidente vascular encefálico. No protocolo proposto o paciente foi formalmente questionado quanto à dificuldade de deglutição, tosse ou falta de ar relacionadas à alimentação e, ainda assim, não foi possível estabelecer correlação entre estas queixas e a presença de alterações, tanto na fase oral (ejeção) quanto na fase faríngea (penetração/aspiração) ${ }^{(17)}$.

A fase de preparo e qualificação ${ }^{(17)}$ da deglutição, da qual os dentes participam ativamente, influem para uma ejeção eficiente, o que reduz em muito o risco de aspiração. Na população estudada, a identificação do comprometimento da arcada dentária mostrou correlação com o risco de aspiração, evidenciando que este fato, que primariamente não guarda relação de causa e efeito com o AVE, acrescenta risco ao quadro do paciente. Este fato pode ser atribuído apenas ao envelhecimento da população, como descrito por Kendall e Leo$\operatorname{nard}^{(14)}$, já que a idade média de nossa população é de $60,95 \pm 13,01$ anos, e é fato notório que o aumento da idade interfere com a qualidade da dentição.

A mobilidade da musculatura da face é importante na pressurização do estojo oral para a ejeção ${ }^{(\mathbf{1 7 )}}$. Seu comprometimento (notadamente a flacidez) faz com que existam escapes e relações organização/ejeção anômalas. Encontramos forte relação entre este comprometimento e a ocorrência de penetração/aspiração de líquido. Isto fortalece a tese de que existe forte influência da fase oral na fase faríngea da deglutição.

A sensibilidade e mobilidade da face dependem dos nervos responsáveis pelas informações qualitativas e quantitativas a respeito do alimento ingerido ${ }^{(\mathbf{1 7 )}}$. Essas informações são a base para uma resposta motora proporcional e adequada. Com seu comprometimento, a fase de qualificação e preparo da deglutição não se processa de maneira adequada, resultando em escape e/ou ejeção ineficiente, o que resulta, também, em alterações da fase faríngea, conforme demonstrou a correlação encontrada no presente estudo, com penetração/aspiração de líquidos.

A língua funciona como base para a organização do alimento e como êmbolo 


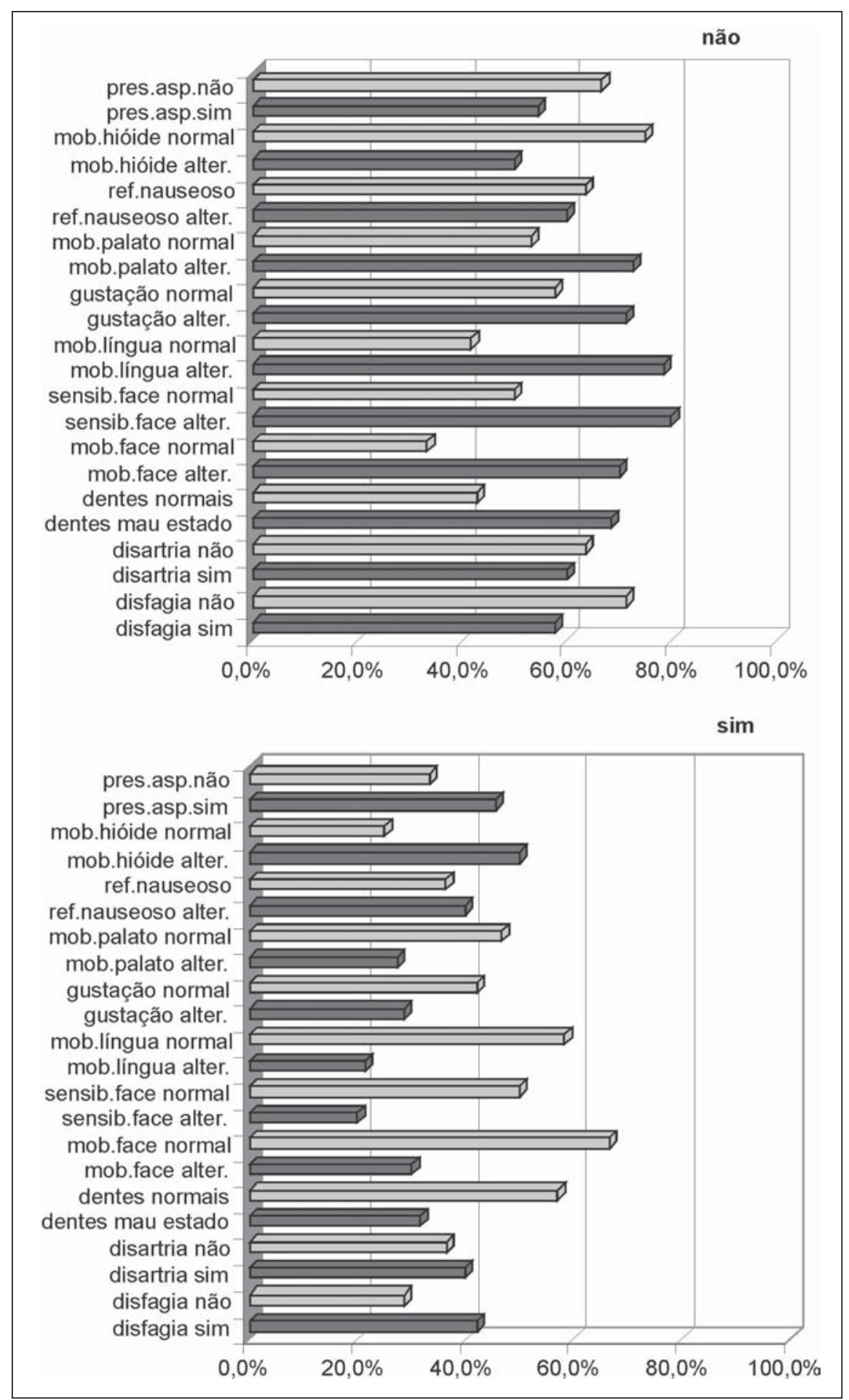

Figura 1. Correlação entre parâmetros clínicos e presença de penetração/aspiração de líquido na videofluoroscopia.

de pressurização na ejeção ${ }^{(17)}$. O comprometimento de sua função motora, principalmente no que diz respeito à coordenação dos movimentos com as outras estruturas envolvidas, causa diminuição de pressão na ejeção, o que compromete sua efi- deste estudo, e apesar de não termos medido formalmente o tempo de preparo oral, este se mostrou alargado em todo o grupo, concordando com os dados de pesquisa de metodologia semelhante ${ }^{(\mathbf{1 8})}$.

O reflexo nauseoso é um reflexo protetor que integra as informações táteis da orofaringe com sua resposta motora ${ }^{(\mathbf{1 7})}$. A observação de sua simples presença, sem qualquer consideração qualitativa, não mostrou correlação com o risco de penetração/aspiração em nossa amostra. Outros grupos, estudando pela videofluoroscopia pacientes com AVE em fase aguda, encontraram correlação entre o risco de aspiração e a presença do reflexo nauseoso e o movimento velar ${ }^{(\mathbf{1 9})}$. Como os nossos pacientes tiveram tempo médio de instalação da incapacidade de 104 dias, já se encontrava em fase de adaptação, como já descrito por Smithard et al. ${ }^{(10)}$.

A mobilidade do hióide é um sinal clínico indireto da abertura em amplitude e duração da transição faringoesofágica, já que a faringe é tracionada e elevada pelos músculos que se inserem neste osso ${ }^{(17)}$. A observação externa deste movimento não foi suficiente para mostrar correlação com o risco de penetração/aspiração nos pacientes estudados.

$\mathrm{Na}$ avaliação clínica, a presença de penetração/aspiração é observada pela ocorrência de disfonia após deglutição de líquido (água). Na avaliação videofluoroscópica este evento foi observado com a ingestão de solução padrão de bário, que tem outras características de sabor e densidade, aproximando-se mais da consistência do iogurte do que da água. Neste estudo, não pudemos observar correlação entre os dois eventos. Acreditamos que esta diferença de características físico-químicas do meio de contraste utilizado tenha reduzido a ocorrência de penetração/aspiração na videofluoroscopia, diminuindo a possibilidade de correlação entre os eventos.

Os estudos de Gordon et al. ${ }^{(6)}$, Smithard et al. ${ }^{(12)}$, Kidd et al. ${ }^{(20)}$, McCullough et al. ${ }^{(21)} \mathrm{e}$ Noll et al. ${ }^{(22)}$ utilizaram volumes predeterminados de bolo, tanto na avaliação clínica quanto videofluoroscópica. A metodologia adotada neste nosso estudo não fixou um volume do bolo a ser deglutido, tanto no exame clínico quanto no videofluoroscópico, ou seja, permitiu que o 
paciente fizesse uso de um dos mecanismos descritos como "adaptação" oral: a redução do volume do bolo. O tempo de preparo oral também ficou sujeito a análise subjetiva, já que sua medição objetiva dependeria da prefixação de um volume uniforme para toda a amostra.

\section{CONCLUSÃO}

Não é seguro predizer a presença de penetração/aspiração de líquidos pela avaliação clínica.

Foi possível estabelecer correlação entre os parâmetros clínicos relacionados à fase oral da deglutição e a presença de penetração/aspiração de líquidos na videofluoroscopia.

Os parâmetros clínicos relacionados à fase faríngea não são correlacionáveis aos achados da videofluoroscopia.

\section{Recomendações}

Todos os pacientes vítimas de AVE devem ter a função de deglutição formalmente avaliada, mesmo sem referir queixas de disfagia, antes de se iniciar a alimentação por via oral.

O protocolo utilizado permitiu identificar alterações da fase oral relacionadas ao risco aumentado de penetração/aspiração.

Como não foi possível identificar fatores de risco para a ocorrência de penetração/aspiração de líquidos, devemos recomendar que toda vítima de AVE seja submetida a avaliação clínica minuciosa e a videofluoroscopia. Se identificadas alterações importantes da fase oral ao exame clínico, a indicação de alimentação por via oral deve ser adiada até a realização do exame de imagem, já que o risco se eleva significativamente.
Como não identificamos qualquer caso de penetração/aspiração de alimentos ou contraste nas consistências pastosa e sólida no grupo estudado, podemos recomendar que pacientes em fase subaguda de instalação de AVE devam ser alimentados preferencialmente com alimentos de consistência pastosa a sólida.

Finalmente, reforçamos o conceito de interdisciplinaridade, que deve nortear a abordagem a pacientes portadores de múltiplas incapacidades, e que sem rotinas terapêuticas previamente e acordadas essa abordagem é inexeqüível.

\section{REFERÊNCIAS}

1. Odderson IR, Keaton JC, McKenna BS. Swallow management in patients on an acute stroke pathway: quality is cost effective. Arch Phys Med Rehabil 1995;76:1130-3.

2. Roth EJ, Harvey RL. Rehabilitation of stroke syndromes. In: Braddom RL, ed. Physical medicine \& rehabilitation. 2nd ed. Philadelphia, PA: Saunders, 2000:1117-60

3. Lawrence ES, Coshall C, Dundas R, et al. Estimates of the prevalence of acute stroke impairments and disability in a multiethnic population. Stroke 2001; 32:1279-84.

4. Aviv JE, Sacco RL, Thomson J, et al. Silent laryngopharyngeal sensory deficits after stroke. Ann Otol Rhinol Laryngol 1997;106:87-93.

5. Daniels SK, Brailey K, Priestly DH, Herrington LR, Weisberg LA, Foundas AL. Aspiration in patients with acute stroke. Arch Phys Med Rehabil 1998;79: 14-9.

6. Gordon M, Clarke I, Cruickshank E, Clifford M, Lamb A, Peck D. Management of patients with stroke III: identification and management of dysphagia. Scottish Intercollegiate Guidelines Network, Nov 1997. Disponível em http://www.sign.ac.uk/ guidelines/published. Acessado em 17/6/2002.

7. Teramoto S, Fukuchi Y. Detection of aspiration and swallowing disorder in older stroke patients: simple swallowing provocation test versus water swallowing test. Arch Phys Med Rehabil 2000;81:1517-9.

8. Costa MMB. Uso de bolo contrastado sólido, líquido e pastoso no estudo videofluoroscópico da dinâmica da deglutição. Radiol Bras 1996;29:35-9.

9. Monteiro JS. Sistema de registro e recuperação de exames da dinâmica da deglutição. (Dissertação de
Mestrado). Rio de Janeiro: Universidade Federal do Rio de Janeiro, 2002.

10. Smithard DG, O'Neill PA, England RE, et al. The natural history of dysphagia following a stroke. Dysphagia 1997;12:188-93.

11. Macedo Fo ED. Estudo comparativo entre a videoendoscopia da deglutição (VED) e a videofluoroscopia (VFC) na avaliação da disfagia orofaríngea pós-acidente vascular cerebral (AVC). (Tese de Doutorado). Curitiba: Universidade Federal do Paraná, 2001.

12. Smithard DG, O'Neill PA, Park C, Morris J. Complications and outcome after acute stroke. Does dysphagia matter? Stroke 1996;27:1200-4.

13. Daniels SK, Ballo LA, Mahoney MC, Foundas AL. Clinical predictors of dysphagia and aspiration risk: outcome measures in acute stroke patients. Arch Phys Med Rehabil 2000;81:1030-3.

14. Kendall KA, Leonard RJ. Bolus transit and airway protection coordination in older dysphagic patients. Laryngoscope 2001;111:2017-21.

15. U.S.Department of Health and Human Services, Agency for Health Care Policy and Research. Diagnosis and treatment of swallowing disorders (dysphagia) in acute-care stroke patients. AHCPR 1999; E023. Disponível em http://www.medscape.com/ govmt/AHCPR/1999/guidelines/AHCPR. dysphsum/AHCPR.dysphsum-toc.htm. Acessado em 20/5/2000.

16. Hamdy S, Aziz Q, Rothwell JC, et al. The cortical topography of human swallowing musculature in health and disease. Nat Med 1996;2:1217-24.

17. Costa MMB. Dinâmica da deglutição: fases oral e faríngea. In: I Colóquio Multidisciplinar de Deglutição e Disfagia do Rio de Janeiro. Rio de Janeiro: PAEDD - Programa Avançado de Estudo da Deglutição e Disfagia, 1998:1-11.

18. Martin BJW, Corlew MM, Wood H, et al. The association of swallowing dysfunction and aspiration pneumonia. Dysphagia 1994;9:1-6.

19. Leder SB. Videofluroscopic evaluation of aspiration with visual examination of the gag reflex and velar movement. Dysphagia 1997;12:21-3.

20. Kidd D, Lawson J, Nesbitt R, MacMahon J. Aspiration in acute stroke: a clinical study with videofluoroscopy. Q J Med 1993;86:825-9.

21. McCullough GH, Wertz RT, Rosenbek JC. Sensitivity and specificity of clinical/bedside examination signs for detecting aspiration in adults subsequent to stroke. J Commun Disord 2001;34:55-72.

22. Noll SF, Bender CE, Nelson MC. Rehabilitation of patients with swalowing disorders. In: Braddom RL, ed. Physical medicine \& rehabilitation. 2nd ed. Philadelphia, PA: Saunders, 2000:535-60. 